Submitted to: Autism Research

Word count: 2160 words

Submission date: 24 June 2019

Title (Commentary):

\title{
Self-processing in individuals with Autism Spectrum Disorder
}

\author{
Running head: \\ Self-processing in Autism Spectrum Disorder \\ Annabel Dineke Nijhof ${ }^{1} \&$ Geoffrey Bird ${ }^{1,2}$
}

${ }^{1}$ Social, Genetic and Developmental Psychiatry Centre, Institute of Psychiatry, Psychology and Neuroscience, King's College London

${ }^{2}$ Department of Experimental Psychology, University of Oxford

*Corresponding author: Annabel.Nijhof@kcl.ac.uk

$$
\text { + } 44(0) 2078480936
$$

Social, Genetic and Developmental Psychiatry Centre

Institute of Psychiatry, Psychology and Neuroscience - PO80

De Crespigny Park,

Denmark Hill, London,

United Kingdom, SE5 8AF 
Acknowledgements: Annabel D. Nijhof is supported by a Newton International Fellowship awarded by the Royal Society. Geoffrey Bird is supported by the Baily Thomas Charitable Trust.

Lay summary ( 60 - 80 words): Research has indicated that individuals with Autism Spectrum Disorder show differences in the processing of self-relevant information. However, as yet, exactly how self-processing differs in autism remains unknown. To further our understanding of the self in autism, future studies should focus on the relationship between different aspects of self-processing, investigating brain activity as well as behaviour, across a wide range of ages.

\begin{abstract}
Research attempting to explain the social difficulties observed in Autism Spectrum Disorder has focused predominantly on difficulties understanding others, but there are indications that self-referential processing is also atypical in autism. For example, infants who later get an autism diagnosis show a reduced response when hearing their own name. In addition, research suggests that the self-bias (the tendency to preferentially process information when self-relevant) is smaller or absent in autism. However, findings are mixed: researchers are yet to clarify exactly those aspects of selfprocessing which are atypical in autism, and in what way they are atypical. To gain further insight into these issues, future studies should focus on whether and how different aspects of self-processing are related in both neurotypical and autistic individuals. Furthermore, the (a)typical development of different aspects of the self, and the impact of the self on different domains of cognitive processing, deserves further attention, requiring studies with participants in a wide age range. Finally, the use of neural measures of self-processing will be invaluable, given the recent hypothesis that autistic individuals may learn to compensate for difficulties by relying on neural pathways which differ from those utilised by neurotypical individuals.
\end{abstract}

Key words: Self-processing; Egocentric bias; Compensation; Social Cognition 
In processing the world around us, humans are biased to preferentially process information with a high degree of relevance to the self. This increased tendency to notice, learn and remember information when it is self-related is referred to as self-bias (Cunningham \& Turk, 2017). Somewhat counter-intuitively, the degree to which individuals preferentially process self-relevant information is likely to impact their social functioning in various ways. For example, a strong bias to process self-relevant cues is likely to lead to a more accurate, comprehensive and earlier-developing model of the self. It has been argued that an accurate model of the self aids understanding of others through the ability to simulate their state in the self. Such a simulation allows one to determine which intentions, emotions, or mental states would be experienced if one was in the other's state and, in turn, these are attributed to the other (Goldman, 2006). Consistent with this view, research indicates that the more accurately individuals can perceive the physiological condition of their own body through interoception, the better understanding they will have of others' emotions (Shah, Catmur, \& Bird, 2017). Similarly, a better understanding of the self may support the representation of other people's minds (Conway, Catmur, \& Bird, 2019) and mental states (Mitchell, 2009).

However, a complete focus on the self at the detriment of processing other-related information is likely to have a negative impact on the development of bidirectional associative links between cues to particular states in others and the corresponding states in the self. These links have been argued to be crucial in the development of imitation (Catmur, Walsh, \& Heyes, 2009; Cook, Bird, Catmur, Press, \& Heyes, 2014), empathy (Bird \& Viding, 2014; Heyes \& Bird, 2007; Quattrocki \& Friston, 2014), and Theory of Mind (Ondobaka, Kilner, \& Friston, 2017). For example, it has been suggested that another's pain comes to be able to invoke pain in the self via the development of bidirectional associations between one's own pain and pained expressions produced by one's caregivers in infancy. Following development of such bidirectional associations, sight of another's pained expression activates a representation of pain in oneself (Heyes \& Bird, 2007). An extreme self-focus may impede the development of such bidirectional associations, and therefore individuals who have an extreme self-bias are likely to exhibit impaired understanding of, and atypical responses to, others (Birch \& Bloom, 2004). Such an extreme focus on the self (or extreme 'egocentrism') has been argued to be a central feature of Autism Spectrum Disorder (Frith \& de Vignemont, 2005), although many studies in fact suggest the opposite 
by demonstrating a reduced self-bias in autism ${ }^{1}$ (e.g. Lombardo \& Baron-Cohen, 2010). Difficulties with social communication and interaction are key characteristics of autism, and atypical self-processing (increased egocentrism as well as impaired self-referential cognition) may provide an important window into these difficulties (Huang et al., 2017; Lombardo et al., 2009). Despite this potential opportunity, self-processing is a relatively understudied area in the autism literature. In this Commentary we point out several aspects of the study of the self in autism that we believe deserve further attention.

Notably, cognitive theories attempting to explain the social difficulties observed in autistic individuals have predominantly focused on challenges in understanding others. The focus on other-related processing in autism is likely to mean that potential differences in selfrelated processing have largely been overlooked; including the potential for such self-related differences to explain social difficulties. This situation is surprising, given that differences in self-processing have long been considered characteristic of autism: one of the earliest and strongest predictors of a later autism diagnosis is an absent or diminished response to hearing one's own name in infancy (Werner, Dawson, Osterling, \& Dinno, 2000), which may be a product of factors such as slower name learning or a weaker association between one's own name and social reward. As this difference in self-processing can be observed even before the first year of age, and with recent studies indicating that self-processing differences may be observed in adults as well as children with autism (Lombardo \& Baron-Cohen, 2010; Uddin, 2011; Williams, 2010), it is also surprising that self-processing has rarely been studied across different age groups of autistic and neurotypical individuals. The human self-concept is known to change throughout development (Harter, 1999), with visual self-recognition and personal pronoun use emerging around the second year of life (Lewis \& Ramsay, 2004), followed by increasing metacognitive self-awareness (Rochat, 2003) and the continuous development of brain regions supporting self-related processing throughout adolescence (Sebastian, Burnett, \& Blakemore, 2008). Little is known, however, about this developmental trajectory in individuals with autism.

Studies in neurotypical participants have revealed that a self-bias is present not only for stimuli that have long been associated with the self (e.g., one's own name), but can also be observed for neutral stimuli that have only recently been associated with the self. For example, on the 'shape-label matching task' (Sui, He, \& Humphreys, 2012) participants learn

\footnotetext{
${ }^{1}$ To respect the wishes of autistic individuals and to use standard scientific parlance, we use language preferred by clinical professionals (e.g., 'individuals with autism'), as well as the term 'autistic', a term endorsed by many individuals with ASD (Kenny et al., 2016).
} 
to differentially associate geometric shapes with themselves or other people. When subsequently tested on these associations, they are faster and more accurate when presented with stimuli associated with the self. A further example is the self-reference effect in memory (Symons \& Johnson, 1997): the term given to the finding that self-relevant material is remembered better than other-relevant material. This effect has been found for stimuli that allow elaborate self-related processing (e.g., personality traits which have been evaluated for the extent to which they describe oneself), but also for random objects after it has been stated they are owned by the participant - the 'ownership effect' (Cunningham, Turk, Macdonald, \& Macrae, 2008). Interestingly, both ownership and trait self-reference effects are reported to be diminished in autism, in children (Gillespie-Smith, Ballantyne, Branigan, Turk, \& Cunningham, 2018) as well as in adults (Grisdale, Lind, Eacott, \& Williams, 2014; Lombardo, Barnes, Wheelwright, \& Baron-Cohen, 2007), suggestive of a decreased self-bias. Other aspects of self-processing, however, seem unaffected in autistic individuals, with typical performance observed for autistic adults on the shape-label matching task (Williams, Nicholson, \& Grainger, 2017), an intact ability to monitor and recall one's own actions in both children and adults with autism (Grainger, Williams, \& Lind, 2014b; Williams \& Happé, 2009), and typical mirror self-recognition in children (Dawson \& McKissick, 1984).

These mixed findings have prompted the development of cognitive models of selfrelated processing in autism, to explain the observed pattern of typical and atypical selfprocessing. For example, several authors have argued that extant findings support models proposing an intact physical self (awareness of one's body and actions), but an altered psychological self, in autism (Elmose, 2016; Uddin, 2011; Williams, 2010). Support for the dissociation of physical and psychological aspects of the self comes from neuroimaging studies showing that these distinct aspects of the self are differentially processed at the neural level (Hu et al., 2016). An alternative account (Williams et al., 2017) states that whereas early-stage self-processing may be intact in autism, late-stage 'deep' evaluation of selfrelated information is likely diminished. This deep evaluation is described as second-order representation: instead of simply tagging something as self-related (first-order representation, e.g.: 'self = triangle'), the self becomes the object of one's own thought (second-order, e.g. 'Am I friendly?'). A specific difficulty with second-order self-representations is consistent with metacognitive impairments in autism (Grainger, Williams, \& Lind, 2014a), as metacognition requires a second-order representation of one's own mental states.

Although these models have a large degree of explanatory power, they are unlikely to fully account for self-processing differences in autism. Two recent electro-encephalography 
(EEG) studies found that the typical event-related potential (ERP; electrical changes in brain activity observed in response to a specific event) amplitude differences between seeing one's own face and another person's face was diminished in both autistic children and adults (Cygan, Tacikowski, Ostaszewski, Chojnicka, \& Nowicka, 2014; Gunji, Inagaki, Inoue, Takeshima, \& Kaga, 2009). Similarly, it has been shown that the ERP response to seeing (Cygan et al., 2014) or hearing (Nijhof, Goris, Brass, Dhar, \& Wiersema, 2018) one's own name in autistic adults is diminished as well. In short, both physical (face) and first-order psychological (name) aspects of self-processing were shown to be atypical in autistic individuals in these studies, using paradigms that did not require second-order representation.

Despite plentiful evidence of altered self-related processing in autism, several issues remain unresolved. A particular shortcoming of existing work is that different aspects of selfprocessing have mostly been studied in isolation. This prevents researchers from understanding the relationship between different measures of self-bias; for example, is it the case that those individuals exhibiting a larger self-referential memory effect would also exhibit a larger self-advantage on the shape-label matching task? Additionally, given that autism is a highly heterogeneous disorder and sample sizes are typically small (especially for neuroimaging studies), findings are hard to generalize across the autistic population as well as across different aspects of self-related processing. In fact, as most findings on self-processing in autism currently rely on single studies, it remains to be seen if any findings will replicate in different samples. Future studies can account for these shortcomings in two ways. First, there is a need for studies attempting to replicate previous findings on self-processing in autism, both at the behavioural and at the neural level. This will increase the reliability of conclusions about the presence or absence of group differences. Second, it would be useful to directly compare performance on tasks focusing on different aspects of self-processing to determine whether they rely on overlapping or distinct mechanisms, and whether these mechanisms are affected in large samples of autistic individuals. However, in order to make any inferences about the mechanisms involved, an essential first step will be to conduct a thorough conceptual analysis of the processes each of the tasks taps into. For example, self-bias on the shape-label matching task is traditionally interpreted as a perceptual effect (Sui et al., 2012), but may well have an attentional component as well. A conceptual analysis of the existing measures of self-referential processing will provide the necessary foundation for building theoretical models of typical and atypical self-processing.

Finally, EEG studies suggest that aspects of self-referential processing that appear typical at the behavioural level may be supported by different underlying neural mechanisms 
in individuals with autism (Cygan et al., 2014; Nijhof et al., 2018). Throughout development, autistic individuals may learn to compensate for their atypical neural functioning by relying on neural pathways that are different from their neurotypical peers. It is possible to speculate that compensatory strategies may be more successful in supporting first- than second-order representations of the self, explaining the distinction observed at the behavioural level. Compensation, 'the processes contributing to improved behavioural presentation of a neurodevelopmental disorder, despite persisting core deficit(s) at cognitive and/or neurobiological levels' (p.731, Livingston \& Happé, 2017), has been studied in relation to other-related processing (White, Frith, Rellecke, Al-Noor, \& Gilbert, 2014). Compensation in relation to self-processing, however, has not been extensively explored. Doing so is of paramount importance in order to increase our understanding of both self- and other-related processing in autism. Of note is the fact that one recent study investigated compensation in autism during both self- and other-related processing (Lai et al., 2018). When compared to neurotypicals and autistic men, autistic women, who 'camouflage' (try to act in a neurotypical manner) more than men, showed a different neural response during selfrepresentation specifically. This is the first indication that compensation plays a role in selfprocessing, as well as other-processing, in autism. These data underscore that neural measures are invaluable for uncovering how individuals with and without autism process self-relevant information. In addition, as compensatory strategies in ASD may only be developed and/or utilised at later ages, this finding also emphasises the need for studies across development.

In conclusion, future research should further scrutinise self-related processing in autism. By employing comprehensive task batteries, using behavioural and neural measures, and adopting a developmental perspective, this research would provide a more complete understanding of the self-related and social difficulties observed in autism. Beyond social difficulties, understanding self-related processing and self-bias in autism may also shed light on other processes thought to rely on a model of the self which have been claimed to be atypical in autism, such as metacognition (Grainger et al., 2014a), a sense of agency (Zalla, Miele, Leboyer, \& Metcalfe, 2015), categorisation based on in-group or out-group membership (Skorich, Gash, Stalker, Zheng, \& Haslam, 2017), and the ability to regulate one's emotions (Mazefsky et al., 2013; Samson, Huber, \& Gross, 2012). 


\section{References}

Birch, S. A. J., \& Bloom, P. (2004). Understanding children's and adults' limitations in mental state reasoning. Trends in Cognitive Sciences, 8(6), 4-9. https://doi.org/10.1016/j.tics.2004.04.011

Bird, G., \& Viding, E. (2014). The self to other model of empathy: Providing a new framework for understanding empathy impairments in psychopathy, autism, and alexithymia. Neuroscience and Biobehavioral Reviews, 47, 520-532. https://doi.org/10.1016/j.neubiorev.2014.09.021

Catmur, C., Walsh, V., \& Heyes, C. (2009). Associative sequence learning: the role of experience in the development of imitation and the mirror system. Philosophical Transactions of the Royal Society B, 364, 2369-2380. https://doi.org/10.1098/rstb.2009.0048

Conway, J. R., Catmur, C., \& Bird, G. (2019). Understanding individual differences in theory of mind via representation of minds, not mental states. Psychonomic Bulletin and Review, 1-15. https://doi.org/https://doi.org/10.3758/s13423-018-1559-x

Cook, R., Bird, G., Catmur, C., Press, C., \& Heyes, C. (2014). Mirror neurons: From origin to function. Behavioral and Brain Sciences, 37(2), 177-192. https://doi.org/10.1017/S0140525X13000903

Cunningham, S. J., \& Turk, D. J. (2017). Editorial: A review of self-processing biases in cognition. Quarterly Journal of Experimental Psychology, 70(6), 987-995. https://doi.org/10.1080/17470218.2016.1276609

Cunningham, S. J., Turk, D. J., Macdonald, L. M., \& Macrae, C. N. (2008). Yours or mine? Ownership and memory. Consciousness and Cognition, 17, 312-318. https://doi.org/10.1016/j.concog.2007.04.003

Cygan, H. B., Tacikowski, P., Ostaszewski, P., Chojnicka, I., \& Nowicka, A. (2014). Neural Correlates of Own Name and Own Face Detection in Autism Spectrum Disorder. PLoS ONE, 9(1), e86020. https://doi.org/10.1371/journal.pone.0086020

Dawson, G., \& McKissick, F. C. (1984). Self-recognition in autistic children. Journal of Autism and Developmental Disorders, 14(4), 383-394. https://doi.org/10.1007/BF02409829

Elmose, M. (2016). A conceptual framework for understanding characteristics of selfawareness associated with autism spectrum disorder. Scandinavian Journal of Child and Adolescent Psychiatry and Psychology, 4(3), 109-114. https://doi.org/10.21307/sjcapp2016-017 
Frith, U., \& de Vignemont, F. (2005). Egocentrism, allocentrism, and Asperger syndrome. Consciousness and Cognition, 14, 719-738. https://doi.org/10.1016/j.concog.2005.04.006

Gillespie-Smith, K., Ballantyne, C., Branigan, H. P., Turk, D. J., \& Cunningham, S. J. (2018). The I in autism: Severity and social functioning in autism are related to selfprocessing. British Journal of Developmental Psychology, 36(1), 127-141. https://doi.org/10.1111/bjdp.12219

Goldman, A. I. (2006). Simulating minds: The philosophy, psychology, and neuroscience of mind reading. New York, NY: Oxford University Press.

Grainger, C., Williams, D. M., \& Lind, S. E. (2014a). Metacognition, metamemory, and mindreading in high-functioning adults with autism spectrum disorder. Journal of Abnormal Psychology, 123(3), 650-659. https://doi.org/10.1037/a0036531

Grainger, C., Williams, D. M., \& Lind, S. E. (2014b). Online action monitoring and memory for self-performed actions in autism spectrum disorder. Journal of Autism and Developmental Disorders, 44(5), 1193-1206. https://doi.org/10.1007/s10803-013-19874

Grisdale, E., Lind, S. E., Eacott, M. J., \& Williams, D. M. (2014). Self-referential memory in autism spectrum disorder and typical development : Exploring the ownership effect. Consciousness and Cognition, 30, 133-141. https://doi.org/10.1016/j.concog.2014.08.023

Gunji, A., Inagaki, M., Inoue, Y., Takeshima, Y., \& Kaga, M. (2009). Event-related potentials of self-face recognition in children with pervasive developmental disorders. Brain and Development, 31(2), 139-147. https://doi.org/10.1016/j.braindev.2008.04.011 Harter, S. (1999). The construction of the self: A developmental perspective. New York: Guilford Press.

Heyes, C., \& Bird, G. (2007). Mirroring, association, and the correspondence problem. In P. Haggard, Y. Rossetti, \& M. Kawato (Eds.), Sensorimotor foundations of higher cognition, attention \& performance XX. Oxford, United Kingdom: Oxford University Press. https://doi.org/10.1093/acprof:oso/9780199231447.003.0021

Hu, C., Di, X., Eickhoff, S. B., Zhang, M., Peng, K., Guo, H., \& Sui, J. (2016). Distinct and common aspects of physical and psychological self-representation in the brain: A metaanalysis of self-bias in facial and self-referential judgements. Neuroscience and Biobehavioral Reviews, 61, 197-207. https://doi.org/10.1016/j.neubiorev.2015.12.003 Huang, A. X., Hughes, T. L., Sutton, L. R., Lawrence, M., Chen, X., Ji, Z., \& Zeleke, W. 
(2017). Understanding the self in individuals with Autism Spectrum Disorders (ASD): A review of literature. Frontiers in Psychology, 8(AUG), 1-8.

https://doi.org/10.3389/fpsyg.2017.01422

Kenny, L., Hattersley, C., Molins, B., Buckley, C., Povey, C., \& Pellicano, E. (2016). Which terms should be used to describe autism? Perspectives from the UK autism community. Autism, 20(4), 442-462. https://doi.org/10.1177/1362361315588200

Lai, M.-C., Lombardo, M. V, Chakrabarti, B., Ruigrok, A. N., Bullmore, E. T., Suckling, J., ... Williams, S. C. (2018). Neural self-representation in autistic women and association with "compensatory camouflaging." Autism, 136236131880715. https://doi.org/10.1177/1362361318807159

Lewis, M., \& Ramsay, D. (2004). Development of Self-Recognition, Personal Pronoun Use, and Pretend Play During the 2nd Year. Child Development, 75(6), 1821-1831.

Livingston, L. A., \& Happé, F. (2017). Conceptualising compensation in neurodevelopmental disorders: Reflections from autism spectrum disorder. Neuroscience and Biobehavioral Reviews, 80(March), 729-742. https://doi.org/10.1016/j.neubiorev.2017.06.005

Lombardo, M. V., Barnes, J. L., Wheelwright, S. J., \& Baron-Cohen, S. (2007). Selfreferential cognition and empathy in autism. PLoS ONE, 2(9), e883. https://doi.org/10.1371/journal.pone.0000883

Lombardo, M. V., \& Baron-Cohen, S. (2010). Unraveling the paradox of the autistic self. Wiley Interdisciplinary Reviews: Cognitive Science, 1(3), 393-403. https://doi.org/10.1002/wcs.45

Lombardo, M. V., Chakrabarti, B., Bullmore, E. T., Sadek, S. A., Pasco, G., Wheelwright, S. J., ... Baron-Cohen, S. (2009). Atypical neural self-representation in autism. Brain, 133(2), 611-624. https://doi.org/10.1093/brain/awp306

Mazefsky, C. A., Herrington, J., Siegel, M., Scarpa, A., Maddox, B. B., Scahill, L., \& White, S. W. (2013). The Role of Emotion Regulation in Autism Spectrum Disorder. Journal of the American Academy of Child \& Adolescent Psychiatry, 52(7), 679-688. https://doi.org/https://doi.org/10.1016/j.jaac.2013.05.006

Mitchell, J. P. (2009). Inferences about mental states. Philosophical Transactions of the Royal Society B, 364, 1309-1316. https://doi.org/10.1098/rstb.2008.0318

Nijhof, A. D., Goris, J., Brass, M., Dhar, M., \& Wiersema, J. R. (2018). Atypical neural responding to hearing one's own name in adults with ASD. Journal of Abnormal Psychology, 127(1), 129-138. https://doi.org/10.1037/abn0000329

Ondobaka, S., Kilner, J., \& Friston, K. (2017). The role of interoceptive inference in theory 
of mind. Brain and Cognition, 112, 64-68. https://doi.org/10.1016/j.bandc.2015.08.002

Quattrocki, E., \& Friston, K. (2014). Autism, oxytocin and interoception. Neuroscience and Biobehavioral Reviews, 47, 410-430.

https://doi.org/http://dx.doi.org/10.1016/j.neubiorev.2014.09.012

Rochat, P. (2003). Five levels of self-awareness as they unfold early in life. Consciousness and Cognition, 12(4), 717-731. https://doi.org/10.1016/S1053-8100(03)00081-3

Samson, A. C., Huber, O., \& Gross, J. J. (2012). Emotion regulation in Asperger's syndrome and high-functioning autism. Emotion, 12(4), 659-665.

https://doi.org/10.1037/a0027975

Sebastian, C., Burnett, S., \& Blakemore, S. (2008). Development of the self-concept during adolescence. Trends in Cognitive Sciences, 12(11), 441-446.

https://doi.org/10.1016/j.tics.2008.07.008

Shah, P., Catmur, C., \& Bird, G. (2017). From heart to mind: Linking interoception, emotion, and theory of mind. Cortex, 93, 220-223.

https://doi.org/http://dx.doi.org/10.1016/j.cortex.2017.02.010

Skorich, D. P., Gash, T. B., Stalker, K. L., Zheng, L., \& Haslam, S. A. (2017). Exploring the Cognitive Foundations of the Shared Attention Mechanism: Evidence for a Relationship Between Self-Categorization and Shared Attention Across the Autism Spectrum.

Journal of Autism and Developmental Disorders, 47(5), 1341-1353.

https://doi.org/10.1007/s10803-017-3049-9

Sui, J., He, X., \& Humphreys, G. W. (2012). Perceptual effects of social salience: Evidence from self-prioritization effects on perceptual matching. Journal of Experimental Psychology: Human Perception and Performance, 38(5), 1105-1117. https://doi.org/10.1037/a0029792

Symons, C. S., \& Johnson, B. T. (1997). The Self-Reference Effect in Memory: A MetaAnalysis. Psychological Bulletin, 121(3), 371-394.

Uddin, L. Q. (2011). The self in autism: An emerging view from neuroimaging. Neurocase, 17(3), 201-208. https://doi.org/10.1080/13554794.2010.509320

Werner, E., Dawson, G., Osterling, J., \& Dinno, N. (2000). Brief report: Recognition of autism spectrum disorder before one year of age: A retrospective study based on home videotapes. Journal of Autism and Developmental Disorders, 30(2), 157-162. https://doi.org/10.1023/A:1005463707029

White, S. J., Frith, U., Rellecke, J., Al-Noor, Z., \& Gilbert, S. J. (2014). Autistic adolescents show atypical activation of the brain's mentalizing system even without a prior history 
of mentalizing problems. Neuropsychologia, 56, 17-25.

https://doi.org/10.1016/j.neuropsychologia.2013.12.013

Williams, D. M. (2010). Theory of own mind in autism: Evidence of a specific deficit in selfawareness? Autism, 14(5), 474-494. https://doi.org/10.1177/1362361310366314

Williams, D. M., \& Happé, F. G. E. (2009). Pre-Conceptual Aspects of Self-Awareness in Autism Spectrum Disorder : The Case of Action-Monitoring. Journal of Autism and Developmental Disorders, 39, 251-259. https://doi.org/10.1007/s10803-008-0619-x

Williams, D. M., Nicholson, T., \& Grainger, C. (2017). The Self-Reference Effect on Perception: Undiminished in Adults with Autism and No Relation to Autism Traits. Autism Research, 1-11. https://doi.org/10.1002/aur.1891

Zalla, T., Miele, D., Leboyer, M., \& Metcalfe, J. (2015). Metacognition of agency and theory of mind in adults with high functioning autism. Consciousness and Cognition, 31, 126138. https://doi.org/10.1016/j.concog.2014.11.001 